\title{
INVERSE LIMITS OF INTEGRAL DOMAINS ARISING FROM ITERATED NAGATA COMPOSITION
}

\author{
DAVID E. DOBBS and MARCO FONTANA*
}

\begin{abstract}
By iterating the type of pullback constructions in which $P^{r} V D s$ arise by Nagata composition, we are led to study a class of inverse $\operatorname{limits} A=\lim A_{n}$ of integral domains indexed by N. After identifying the prime spectrum, the localizations, and the integral closure of $A$, we then characterize when, i.a., such (typically infinite-dimensional) $A$ is a Prüfer domain, Bézout domain, divided domain, or $P^{r} V D$.
\end{abstract}

\section{Introduction}

As the literature contained several interesting examples of directed unions of (commutative) integral domains, it was appropriate to study direct limits of integral domains in [10], [9]. The present paper initiates a similar study of inverse limits of integral domains, in part to expand upon examples such as the following (cf. Theorem 2.1 (c) and Corollary 2.7): if $k$ is a field, then

$$
k\left[\left[X_{1}\right]\right]+X_{2} k\left(\left(X_{1}\right)\right)\left[\left[X_{2}\right]\right]+X_{3} k\left(\left(X_{1}, X_{2}\right)\right)\left[\left[X_{3}\right]\right]+\cdots
$$

is a valuation domain. For simplicity (and with an eye on the intended examples), we consider only inverse limits of directed systems indexed by $\mathrm{N}$, the set of positive integers. As [10] was motivated largely by the result that the class of Prüfer domains is stable under direct limit, we begin by establishing the analogous result for inverse limit in the local case. Specifically, Theorem 2.1 (g) states that any inverse limit of valuation domains is a valuation domain. We finally establish a non-local analogue (concerning inverse limits of Prüfer domains) in Theorem 2.21. This result is developed in context of the special type of inverse limit to which this paper is devoted. This context is suggested by iterating the pullback construction of $P^{r} V D s$ in [12, Théorème 1.3], itself a generalization of the so-called Nagata composition of valuation domains [20, page 35].

\footnotetext{
* Supported in part by NATO Collaborative Research Grant No. 970140. Fontana thanks the University of Tennessee for its warm hospitality during his visit in July 1997.

Received April 22, 1998.
} 
Applications of $P^{r} V D s$ are not new: see, for instance, their role in realizing arbitrary dimension sequences in [8] and their implicit role as iterated $D+M$ constructions in the work of Seidenberg [22] on constructing polynomial rings which realize specified Krull dimensions. However, " $P{ }^{\infty} V D s$ " such as the ring displayed above are new, inasmuch as they have infinite Krull dimension. To expand upon this observation, we identify $\operatorname{Spec}(A)$ in Theorem 2.5 (a), where $A$ is the type of inverse limit under consideration here. One eventual upshot is Remark 2.9, which shows how to extend the factorizationtheoretic work in [3] to produce an infinite-dimensional non-fragmented valuation domain with no atoms. To prepare for other applications (such as the above-mentioned result on Prüfer domains), we determine the integral closure of $A$ (in Theorem 2.12) and the localizations of $A$ (in Proposition 2.15 (d)). As additional applications, in the spirit of [10], we characterize when $A$ is a divided domain (in the sense of [5]) in Corollary 2.17, a $P^{r} V D$ in Proposition 2.19, a pseudo-valuation domain (in the sense of [17]) in Corollary 2.20, and a Bézout domain in Corollary 2.23.

If $D$ is an integral domain, we let $\operatorname{dim}(D)$ denote the Krull dimension of $D$, $D^{\prime}$ the integral closure of $D, q f(D)$ the quotient field of $D, \operatorname{Max}(D)$ the set of maximal ideals of $D$, and $\mathscr{U}(D)$ the set of units of $D$. As in [5], $P \in \operatorname{Spec}(D)$ is a divided prime ideal of $D$ if $P D_{P}=P$; this is equivalent to saying that the following diagram of canonical homomorphisms:

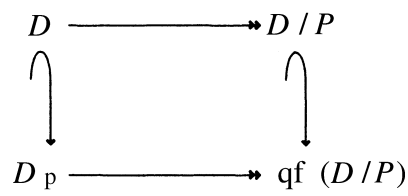

is a pullback. The integral domain $D$ is a divided domain if each $P \in \operatorname{Spec}(D)$ is a divided prime ideal of $D$; and $D$ is a locally divided domain if $D_{P}$ is a divided domain for each $P \in \operatorname{Spec}(D)$. In this paper, the most important examples of divided domains are $P^{r} V D s$ (in particular, (pseudo-)valuation domains), and our most important examples of locally divided domains are Prüfer domains. Following [19, page 28], we denote the properties of lyingover, going-up, going-down and incomparability by LO, GU, GD and INC, respectively. Any unexplained material is standard, as in [15], [19].

After this paper had been drafted, the second-named author gave a talk on it at a meeting held in Fez, Morocco in October 1997. Following the talk, Professor M. Tabaâ mentioned to him that some of our results overlap those of Wiseman [24], a paper with which we had not been acquainted. Although Wiseman's motivations involving linearly compact modules in [24] are different from ours here, one of his contexts [24, page 1109] is a special case of 
ours. As a result, [24] contains special cases of our Theorem 2.1 (d) and Proposition 2.4 (d), as well as special cases of [11, Corollary 1.5 (5)]. Most significantly, in view of Proposition 2.4 (c), whose assertion is a hypothesis for the context of [24], we see that [24, Proposition 3.4] is essentially equivalent to our Theorem 2.12, concerning the commuting of inverse limit and integral closure.

\section{Results}

Recall that direct limit preserves integral domains [16, Proposition 6.1.6 (i)], reduced rings [16, Corollaire 6.1.3], and Prüfer domains [10, Proposition 2.5 (a)]. We begin by establishing some analogues for inverse limit.

TheOREM 2.1. Let $\left(A_{n}, \varphi_{n, m}: A_{n} \rightarrow A_{m} ; n \geq m \geq 1\right)$ be an inverse system of commutative rings, with $\varphi_{n, n}$ taken to be the identity map, and let $A=\lim A_{n}$ be the inverse limit.

Put $\Phi_{n}: A \rightarrow A_{n}$ the canonical map, and $Q_{n}:=\operatorname{ker}\left(\Phi_{n}\right)$. Then

(a) $A=\left\{\left(a_{n}\right) \in \prod A_{n}: \varphi_{n+1, n}\left(a_{n+1}\right)=a_{n}\right.$ for each $\left.n \in \mathbf{N}\right\}$.

(b) For each $n \in \mathrm{N}, \Phi_{n}$ is the composite of the inclusion map $A \hookrightarrow \prod A_{k}$ and the canonical projection $\prod A_{k} \rightarrow A_{n}$.

(c) For each $n \in \mathrm{N}, Q_{n}=\left\{\left(a_{k}\right) \in A: a_{k}=0\right.$ for each $\left.k \leq n\right\}$.

(d) $Q_{1} \supseteq Q_{2} \supseteq Q_{3} \supseteq \ldots$ and $\bigcap Q_{n}=0$.

(e) If $A_{n}$ is an integral domain for each $n$, then $A$ is an integral domain.

(f) If $A_{n}$ is reduced for each $n$, then $A$ is reduced.

(g) If $A_{n}$ is a valuation domain for each $n$, then $A$ is a valuation domain.

Proof. According to the usual construction of inverse limit we may view $A=\left\{\left(a_{n}\right) \in \prod A_{n}: \varphi_{n, m}\left(a_{n}\right)=a_{m}\right.$ whenever $\left.n \geq m\right\}$. Then (a), (b) and (c) are immediate consequences of the definitions. Observe that the additive identity of $A$ is $0=\left(0_{n}\right) \in A$, where $0_{n}$ is the additive identity element of $A_{n}$.

Next, (d) follows from (c).

(e) Deny. Then there exist nonzero $\alpha=\left(\alpha_{n}\right), \beta=\left(\beta_{n}\right) \in A$ such that $\left(\alpha_{n} \beta_{n}\right)=\alpha \beta=0=\left(0_{n}\right) \in A$. As $\alpha \neq 0=\left(0_{n}\right)$, there exists an index $i$ such that $\alpha_{i} \neq 0_{i}$. Similarly, $\beta_{j} \neq 0_{j}$ for some index $j$, since $\beta \neq 0$. Without loss of generality, $i \geq j$. As $\varphi_{i, j}\left(\beta_{i}\right)=\beta_{j} \neq 0_{j}$ and $\varphi_{i, j}$ is a homomorphism, $\beta_{i} \neq 0_{i}$. However, $\alpha_{i} \beta_{i}=0_{i}$, contradicting the hypothesis that $A_{i}$ is an integral domain.

(f) Suppose $\alpha=\left(\alpha_{n}\right) \in A$ is nilpotent. Then for some $\nu \in \mathrm{N},\left(0_{n}\right)=0=$ $\alpha^{\nu}=\left(\alpha_{n}^{\nu}\right)$. Hence, for each $n \in \mathrm{N}, \alpha_{n}^{\nu}=0_{n}$, and so $\alpha_{n}=0_{n}$ since $A_{n}$ is reduced. It follows that $\alpha=\left(0_{n}\right)=0$, whence $A$ is reduced.

(g) Consider nonzero elements $\alpha=\left(\alpha_{n}\right), \beta=\left(\beta_{n}\right) \in A$. By (a), it is enough to show that either $\alpha \in A \beta$ or $\beta \in A \alpha$. 
Case 1: for each $n \in \mathrm{N}, \alpha_{n}=u_{n} \beta_{n}$ for some $u_{n} \in A_{n}$. If $n \geq m$, then

$$
u_{m} \beta_{m}=\alpha_{m}=\varphi_{n, m}\left(\alpha_{n}\right)=\varphi_{n, m}\left(u_{n} \beta_{n}\right)=\varphi_{n, m}\left(u_{n}\right) \varphi_{n, m}\left(\beta_{n}\right)=\varphi_{n, m}\left(u_{n}\right) \beta_{m} .
$$

Since $A_{m}$ is an integral domain, either $\varphi_{n, m}\left(u_{n}\right)=u_{m}$ or $\beta_{m}=0_{m}$. If $\beta_{m} \neq 0_{m}$ (regardless of the choice of $n$ ), then $u:=\left(u_{n}\right) \in A$ and $\alpha=u \beta \in A \beta$. So, without loss of generality, $\beta_{m}=0_{m}$ for some $m \in \mathrm{N}$. Let $k$ be the maximal such $m$. (Note that $k$ exists since $\beta \neq 0$ and $\varphi_{i, j}\left(0_{i}\right)=0_{j}$ whenever $i \geq j$.) By the above reasoning, $\varphi_{n, m}\left(u_{n}\right)=u_{m}$ whenever $k<m \leq n$. Also, if $1 \leq i \leq k$, then $\beta_{i}=\varphi_{k, i}\left(\beta_{k}\right)=\varphi_{k, i}\left(0_{k}\right)=0_{i}$, and so $\alpha_{i}=u_{i} \beta_{i}=0_{i}$. Consider $v=\left(v_{n}\right) \in \prod A_{n}$ defined by

$$
v_{n}= \begin{cases}u_{n} & \text { if } n>k \\ \varphi_{k+1, n}\left(u_{k+1}\right) & \text { if } 1 \leq n \leq k .\end{cases}
$$

Since we have an inverse system, $\varphi_{n, m}\left(v_{n}\right)=v_{m}$ whenever $n \geq m$, whence $v \in A$. Also, $\alpha_{n}=v_{n} \beta_{n}$ for each $n$. (The assertion reduces to $0_{n}=0_{n}$ if $n \leq k$ and to the choice of $u_{n}$ if $n>k$.) Hence, $\alpha=v \beta \in A \beta$.

It remains to consider what happens if Case 1 does not apply. Since each $A_{i}$ is a valuation domain, we are reduced, without loss of generality, to

Case 2: for some $n>m$, there exist $r_{n} \in A_{n}$ and $s_{m} \in A_{m}$ such that $\alpha_{n}=r_{n} \beta_{n}, \quad \beta_{m}=s_{m} \alpha_{m}, \quad \beta_{n} \notin A_{n} \alpha_{n}, \quad$ and $\alpha_{m} \notin A_{m} \beta_{m}$. In particular, $r_{n} \in A_{n} \backslash \mathscr{U}\left(A_{n}\right)$ and $s_{m} \in A_{m} \backslash \mathscr{U}\left(A_{m}\right)$. Then

$\alpha_{m}=\varphi_{n, m}\left(\alpha_{n}\right)=\varphi_{n, m}\left(r_{n} \beta_{n}\right)=\varphi_{n, m}\left(r_{n}\right) \varphi_{n, m}\left(\beta_{n}\right)=\varphi_{n, m}\left(r_{n}\right) \beta_{m}=\varphi_{n, m}\left(r_{n}\right) s_{m} \alpha_{m}$.

Since $s_{m}$ is a nonunit of $A_{m}, \varphi_{n, m}\left(r_{n}\right) s_{m} \neq 1$ and so, since $A_{m}$ is an integral domain, $\alpha_{m}=0_{m}$. This contradict $\alpha_{m} \notin A_{m} \beta_{m}$. Therefore, Case 2 does not occur.

We proceed to fix the riding assumptions and notation for the rest of the paper. We assume given $\left\{A_{1} ;\left(K_{n}, B_{n}\right): n \in \mathrm{N}\right\}$ such that for each $n$ :

- $B_{n}$ is a quasilocal integral domain with maximal ideal $M_{n} \neq 0$;

- $K_{n}=B_{n} / M_{n}, \quad \varphi_{n}: B_{n} \rightarrow K_{n}$ is the canonical surjection and $q f\left(B_{n}\right) \subseteq K_{n+1}$; and

- $A_{1}$ is an integral domain but not a field, $q f\left(A_{1}\right) \subseteq K_{1}$.

For $n>1$, let $A_{n+1}$ be the pullback $A_{n+1}:=B_{n} \times_{K_{n}} A_{n}$. Note that $A_{n+1}$ is canonically contained in $B_{n}$ and so also in $K_{n+1}$. Since $M_{n} \neq 0, A_{n+1}$ and $B_{n}$ share a common nonzero ideal (i.e. $M_{n}$ ), and so $q f\left(A_{n+1}\right)=q f\left(B_{n}\right)$ : cf. [15, page 326]. Thus, the above data lead to the following set of pullback diagrams (reminiscent of the description of $P^{r} V D s$ in the diagram in [12, page 188]): 


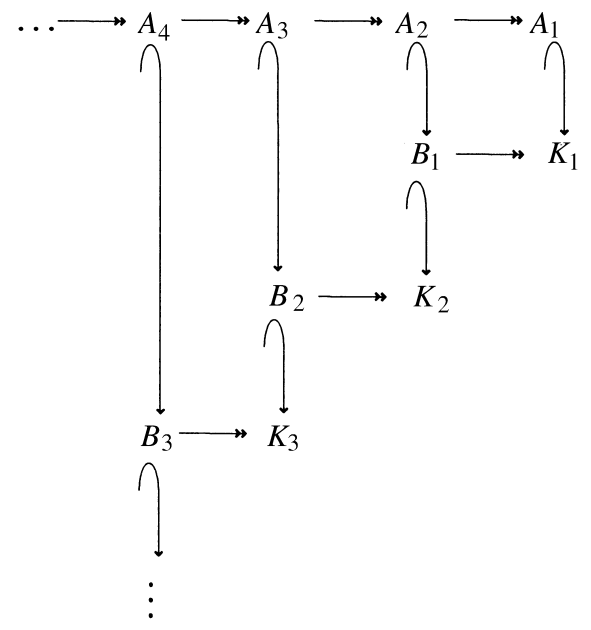

where $\varphi_{n, n-1}: A_{n} \rightarrow A_{n-1}$ is the canonical surjection arising from the fact that $A_{n}=\varphi_{n-1}^{-1}\left(A_{n-1}\right)$ for all $n \geq 2$. If $n>m$, consider the surjection

$$
\varphi_{n, m}=\varphi_{m+1, m} \circ \cdots \circ \varphi_{n, n-1}: A_{n} \rightarrow A_{m},
$$

with $\varphi_{n, n}: A_{n} \rightarrow A_{n}$ taken to be the identity map. The inverse system determined by the homomorphisms $\varphi_{n, m}$ is called the inverse system generated by $\left\{A_{1} ;\left(K_{n}, B_{n}\right): n \in \mathrm{N}\right\}$. Put, as above,

$A:=\lim _{\leftarrow} A_{n}, \quad \Phi_{n}: A \rightarrow A_{n} \quad$ the canonical map, and $Q_{n}:=\operatorname{ker}\left(\Phi_{n}\right)$.

We next collect some basic facts.

LEMmA 2.2. Under the riding assumptions, we have:

(a) For each $n \in \mathrm{N}, \Phi_{n}$ is surjective.

(b) For each $n \in \mathrm{N}, Q_{n} \in \operatorname{Spec}(A)$ and $A / Q_{n} \cong A_{n}$, so $A$ is an integral domain.

(c) For each $n \in \mathrm{N}, M_{n}=\operatorname{ker}\left(\varphi_{n+1, n}\right)$ and $Q_{n}=\Phi_{n+1}^{-1}\left(M_{n}\right)$.

Proof. (a) follows from Theorem 2.1 (b), since each $\varphi_{n, n-1}$ is surjective. Then (b) follows from the First Isomorphism Theorem and Theorem 2.1 (e), since $\Phi_{n}$ is surjective and $A_{n}$ is an integral domain. Finally, for (c), observe that the pullback description of $A_{n+1}$ yields that $\operatorname{ker}\left(\varphi_{n+1, n}\right)=M_{n}$; then, since $\Phi_{n}=\varphi_{n+1, n} \circ \Phi_{n+1}$, we have that $Q_{n}=\operatorname{ker}\left(\Phi_{n}\right)=\Phi_{n+1}^{-1}\left(M_{n}\right)$.

The next result will often permit us to assume, without loss of generality, that $K_{n}=q f\left(A_{n}\right)$ for each $n \in \mathrm{N}$.

Proposition 2.3. For each $n \in \mathrm{N}$, let $K_{n}^{*}=q f\left(A_{n}\right)$ and $B_{n}^{*}=B_{n} \times_{K_{n}} K_{n}^{*}$. Then: 
(a) For each $n \in \mathrm{N}$, there is a canonical isomorphism $A_{n+1} \cong B_{n}^{*} \times_{K_{n}^{*}} A_{n}$.

(b) The inverse system generated by $\left\{A_{1} ;\left(K_{n}^{*}, B_{n}^{*}\right): n \in \mathrm{N}\right\}$ is the same as the inverse system generated by $\left\{A_{1} ;\left(K_{n}, B_{n}\right): n \in \mathrm{N}\right\}$.

Proof. (a) follows from the canonical isomorphism

$$
A_{n+1}=B_{n} \times_{K_{n}} A_{n} \cong\left(B_{n} \times_{K_{n}} K_{n}^{*}\right) \times_{K_{n}^{*}} A_{n}=B_{n}^{*} \times_{K_{n}^{*}} A_{n} .
$$

We thus see that the two inverse systems have the same $\varphi_{n+1, n}$ maps, and (b) follows.

We pause to observe that in Proposition $2.3, B_{n}^{*}$ retains the property of $B_{n}$ of being a quasilocal integral domain with maximal ideal $M_{n}$ (cf. [11, Theorem 1.4, Proposition 2.1 (9)]). More precisely, by [2], $\operatorname{Spec}\left(B_{n}\right)=\operatorname{Spec}\left(B_{n}^{*}\right)$.

We observe also that, given our riding assumptions, $A$ is never a Noetherian ring. As a matter of fact, if $A$ is Noetherian then clearly $A_{n}$ is also Noetherian for each $n \geq 1$ (Lemma 2.2 (b)); but $A_{2}$ is Noetherian if and only if $B_{1}$ is Noetherian, $A_{1}$ is a field and $A_{1} \subset K_{1}$ is a finite (-dimensional) field extension [11, Theorem 2.3].

Finally, we observe that the ring

$$
A=k\left[\left[X_{1}\right]\right]+X_{2} k\left(\left(X_{1}\right)\right)\left[\left[X_{2}\right]\right]+X_{3} k\left(\left(X_{1}, X_{2}\right)\right)\left[\left[X_{3}\right]\right]+\cdots
$$

considered in the introduction, is the inverse limit of the inverse system generated by $\left\{k\left[\left[X_{1}\right]\right],\left(k\left(\left(X_{1}, X_{2}, \ldots, X_{n}\right)\right), k\left(\left(X_{1}, X_{2}, \ldots, X_{n}\right)\right)\left[\left[X_{n+1}\right]\right]\right): n \in \mathrm{N}\right\}$. Since, in this case, $A_{1}=k\left[\left[X_{1}\right]\right]$ and $B_{n}=k\left(\left(X_{1}, \ldots, X_{n}\right)\right)\left[\left[X_{n+1}\right]\right]$ are (discrete) valuation domains, then, by induction on $n \geq 1$ and using Nagata composition, it is easy to see that

$$
A_{n+1}=k\left[\left[X_{1}\right]\right]+X_{2} k\left(\left(X_{1}\right)\right)\left[\left[X_{2}\right]\right]+\cdots+X_{n+1} k\left(\left(X_{1}, X_{2}, \ldots, X_{n}\right)\right)\left[\left[X_{n+1}\right]\right]
$$

is also a valuation domain, for each $n \in \mathrm{N}$. Therefore, by Theorem $2.1(\mathrm{~g}), A$ is a valuation domain.

We proceed to analyze the prime spectrum for the special type of inverse limit being studied here, from which we will deduce, in particular, further properties of our motivating example. For $r \geq n$ in $\mathrm{N}$, put $Q_{r, n}:=\operatorname{ker}\left(\varphi_{r, n}\right)$.

Proposition 2.4.

(a) $Q_{r, n}=\Phi_{r}\left(Q_{n}\right)$ if $r \geq n$ and $Q_{r, n}=\varphi_{r, n+1}^{-1}\left(M_{n}\right)$ if $r \geq n+1$.

(b) For each $n \in \mathrm{N}, \lim _{\leftarrow}\left\{Q_{r, n}: r \geq n\right\}=Q_{n}$.

(c) If $r \geq n$, then $Q_{r, n}$ is a divided prime ideal of $A_{r}$.

(d) For each $n \in \mathrm{N}, Q_{n}$ is a divided prime ideal of $A$.

Proof. (a) As $\varphi_{n, n}$ is an identity map, its kernel, $Q_{n, n}$, is 0 . Of course, $\Phi_{n}\left(Q_{n}\right)$ is also 0 , since $Q_{n}=\operatorname{ker}\left(\Phi_{n}\right)$. Thus, without loss of generality, $r \geq n+1$. As in the proof of Lemma 2.2 (c), $\Phi_{n}=\varphi_{r, n} \circ \Phi_{r}$, whence 
$Q_{n}=\Phi_{n}^{-1}(0)=\Phi_{r}^{-1}\left(\operatorname{ker}\left(\varphi_{r, n}\right)\right)=\Phi_{r}^{-1}\left(Q_{r, n}\right)$. Since $\Phi_{r}$ is surjective, it follows that $\Phi_{r}\left(Q_{n}\right)=Q_{r, n}$. Since $\varphi_{r, n}=\varphi_{n+1, n} \circ \varphi_{r, n+1}$ and $M_{n}=\operatorname{ker}\left(\varphi_{n+1, n}\right)$, we see similarly that $\varphi_{r, n+1}^{-1}\left(M_{n}\right)=\operatorname{ker}\left(\varphi_{r, n}\right)=Q_{r, n}$.

(b) If $r \geq n$, it follows from the surjectivity of $\varphi_{r+1, r}$ and the second assertion in (a) that $\varphi_{r+1, r}$ restricts to a surjection $Q_{r+1, n} \rightarrow Q_{r, n}$. Since $\lim _{\leftarrow}$ preserves monomorphisms, we can view $\lim \left\{Q_{r, n}: r \geq n\right\}$ inside $\lim \left\{A_{r}: r \geq n\right\}$, which, by cofinality, is just $A$. It now follows easily from the above comments that $\lim \left\{Q_{r, n}: r \geq n\right\}=Q_{n}$.

(c) By Proposition 2.3 (b), we may assume that $K_{n}=q f\left(A_{n}\right)$ for each $n \in \mathrm{N}$. Of course, $Q_{r, n}$ is a prime ideal of $A_{r}$, since $\varphi_{r, n}$ is a surjective homomorphism and $A_{n}$ is an integral domain. It remains only to prove the "divided" assertion. This is evident if $r=n$, since $Q_{n, n}=0$. Next, for $r=n+1$, observe that $Q_{n+1, n}=M_{n}$ by (a) and, by a calculation using $K_{n}=q f\left(A_{n}\right)$, we check that $B_{n}=\left(A_{n+1}\right)_{M_{n}}$ (cf. also [14, Lemma 1.1.6]). Then the pullback description of $A_{n+1}$ may be identified with the statement that $A_{n+1}=\left(A_{n+1}\right)_{M_{n}} \times_{q f\left(A_{n+1} / M_{n}\right)}\left(A_{n+1} / M_{n}\right)$; i.e., $Q_{n+1, n}=M_{n}$ is a divided prime ideal of $A_{n+1}$.

It follows easily from the second assertion in (a) that $\varphi_{r+1, r}^{-1}\left(Q_{r, n}\right)=Q_{r+1, n}$. Thus, in view of the pullback description of $A_{n+i}$ for $i \geq 2$, a proof of (c) may be completed (by induction on $r$ ) by proving the following general result.

If $B$ is a quasilocal integral domain with maximal ideal $M$ and residue field $K, \varphi: B \rightarrow K$ the canonical surjection, $D$ an integral domain with quotient field $K$, and $P$ a divided prime ideal of $D$, then $P^{*}:=\varphi^{-1}(P)$ is a divided prime ideal of $D^{*}:=\varphi^{-1}(D)=D \times_{K} B$.

For a proof, observe as above that the condition $K=q f(D)$ ensures that $B=D_{M}^{*}$, whence $M D_{M}^{*}=M$ and, in particular, $M D_{P^{*}}^{*}=M$. As we may identify $D=D^{*} / M$ and $D_{P}=\left(D^{*} / M\right)_{P^{*} / M}=D_{P^{*}}^{*} / M D_{P^{*}}^{*}=D_{P^{*}}^{*} / M$, we have a pullback diagram

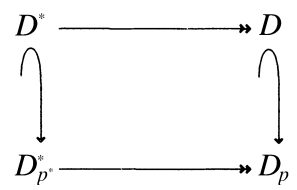

In addition, since $P$ is a divided prime ideal of $D$, there is a pullback diagram

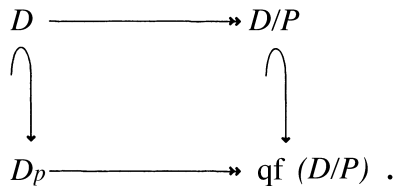


As we may identify $D^{*} / P^{*}=D / P$, juxtaposition of the above diagrams produces a pullback diagram

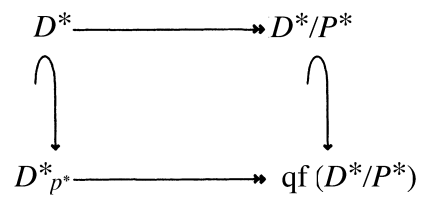

thus showing that $P^{*}$ is divided in $D^{*}$.

(d) We show that if $a=\left(a_{k}\right) \in A \backslash Q_{n}$, then $Q_{n} \subseteq a Q_{n}$. Let $x=\left(x_{k}\right) \in Q_{n}$. Then $x_{r} \in Q_{r, n}$ whenever $r \geq n$, by (b). Also by (b), $a_{r} \in A_{r} \backslash Q_{r, n}$ whenever $r \geq n$. Hence, by (c), if $r \geq n$, there exists $y_{r} \in Q_{r, n}$ such that $x_{r}=a_{r} y_{r}$; moreover, $y_{r}$ is uniquely determined since $a_{r} \neq 0$. Since $\varphi_{r+1, r}\left(x_{r+1}\right)=x_{r}$ and $A_{r}$ is an integral domain, we see easily that $\varphi_{r+1, r}\left(y_{r+1}\right)=y_{r}$ for each $r \geq n$. Moreover, $x_{i}=0$ whenever $i \leq n$, by Theorem 2.1 (c). Since $x_{n}=a_{n} y_{n}$ and $A_{n}$ is an integral domain, $y_{n}=0$. Put $y_{i}=0$ if $1 \leq i<n$ and $y:=\left(y_{k}\right) \in \prod A_{k}$. Observe that $y \in A$ and $x_{k}=a_{k} y_{k}$ for each $k \in \mathrm{N}$. In fact, $y \in Q_{n}$ by (b), and so $x=a y \in a Q_{n}$.

Since $A / Q_{n} \cong A_{n}, \operatorname{Spec}\left(A_{n}\right)$ embeds canonically in $\operatorname{Spec}(A)$. For convenience, we identify $\operatorname{Spec}\left(A_{n}\right)$ with its image in $\operatorname{Spec}(A)$. In particular, $\operatorname{Spec}\left(A_{n}\right) \subseteq \operatorname{Spec}\left(A_{n+1}\right) \subseteq \operatorname{Spec}(A)$. With this convention, the following useful result is easy to state.

THEOREM 2.5 .

(a) $\operatorname{Spec}(A) \backslash\{0\}=\bigcup_{n=1}^{\infty} \operatorname{Spec}\left(A_{n}\right)$.

(b) For each $n \in \mathrm{N}, \operatorname{Max}(A)=\operatorname{Max}\left(A_{n}\right)=\operatorname{Max}\left(A_{1}\right)$.

(c) Let $a=\left(a_{k}\right) \in A$. Then

$$
a \in \mathscr{U}(A) \Leftrightarrow a_{k} \in \mathscr{U}\left(A_{k}\right) \text { for each (resp., some) } k \in \mathrm{N} \Leftrightarrow a_{1} \in \mathscr{U}\left(A_{1}\right) \text {. }
$$

Proof. (a) Since $M_{n} \neq 0$, it follows from Proposition 2.4 (a) that $Q_{n} \neq 0$, and so the zero prime ideal of $A$ is not in (the canonical image of) $\operatorname{Spec}\left(A_{n}\right)$. Therefore, it suffices to show that if $0 \neq P \in \operatorname{Spec}(A)$, then $P \in \operatorname{Spec}\left(A_{k}\right)$ for some $k \in \mathrm{N}$. Now, for each $k$, since $Q_{k}$ is divided (by Proposition 2.4 (d)), either $P \subset Q_{k}$ or $Q_{k} \subseteq P$. As $P \neq 0$, it follows from Theorem 2.1 (d) that $P$ is not contained in $\bigcap Q_{k}$. Hence, there exists $k \in \mathrm{N}$ such that $Q_{k} \subseteq P$. In particular, $P \in \operatorname{Spec}\left(A_{k}\right)$.

(b) Let $n \in \mathrm{N}$. By Proposition 2.4 (d), $Q_{n}$ is contained in each maximal ideal of $A$. Using $A / Q_{n} \cong A_{n}$ and the above convention, we infer $\operatorname{Max}(A)=\operatorname{Max}\left(A / Q_{n}\right)=\operatorname{Max}\left(A_{n}\right)$.

(c) If $M \in \operatorname{Max}(A)$, use the identifications in (b) to view $M$ as 
$M^{(k)} \in \operatorname{Max}\left(A_{k}\right)$ for each $k \in \mathrm{N}$. Since $\Phi_{1}(a)=a_{1}$, we see that $a \in M \Leftrightarrow a_{1} \in M^{(1)}$. Therefore,

$$
\begin{aligned}
a \in \mathscr{U}(A) & \Leftrightarrow a \notin M & \text { for each } & M \in \operatorname{Max}(A) \\
& \Leftrightarrow a_{1} \notin M^{(1)} & \text { for each } & M^{(1)} \in \operatorname{Max}\left(A_{1}\right) \Leftrightarrow a_{1} \in \mathscr{U}\left(A_{1}\right) .
\end{aligned}
$$

Since ring-homomorphisms send units to units, the assertion follows.

Corollary 2.6. $A$ is an integral domain and $\operatorname{dim}(A)=\infty$. Moreover, $A$ is quasilocal if and only if $A_{1}$ is quasilocal.

Proof. The first assertion follows from Theorem 2.1 (e), the third from Theorem 2.5 (b). To show $\operatorname{dim}(A)=\infty$, note that $\operatorname{Spec}\left(A_{n}\right) \neq \operatorname{Spec}\left(A_{n+1}\right)$ since $Q_{n+1, n} \neq 0$.

We next revisit the theme of Theorem 2.1 (g) for the special type of inverse limit under consideration.

COROLlaRy 2.7. The following conditions are equivalent:

(1) For each $n \in \mathrm{N}, A_{n}$ is a valuation domain;

(2) $A_{1}$ is a valuation domain and, for each $n \in \mathrm{N}, B_{n}$ is a valuation domain and $K_{n}=q f\left(A_{n}\right)$;

(3) $A$ is a valuation domain.

Proof. (1) $\Rightarrow$ (3) by Theorem 2.1 (g). Also, (3) $\Rightarrow$ (1) since $A / Q_{n} \cong A_{n}$ and factor domains of valuation domains are valuation domains [15, Proposition $13.2(2)]$.

(1) $\Leftrightarrow(2)$ : It follows from [15, Theorem 26.1 (1)] that if $A_{n+1}$ is a valuation domain, then $K_{n}=q f\left(A_{n}\right)$. Therefore, applying [11, Theorem 2.4 (1)] to the pullback $A_{n+1}=B_{n} \times_{K_{n}} A_{n}$ leads to the following result: $A_{n+1}$ is a valuation domain if and only if $B_{n}$ and $A_{n}$ are valuation domains and $K_{n}=q f\left(A_{n}\right)$. Using this result repeatedly, we obtain both $(1) \Rightarrow(2)$ and $(2) \Rightarrow(1)$.

Before addressing integral closure, we settle (in Remark 2.9) one of the motivating questions mentioned in the Introduction. To this end, we need only the following sortie into factorization theory.

Proposition 2.8. If $A_{n}$ has no atoms (i.e., irreducible elements) for each $n \in \mathrm{N}$, then $A$ has no atoms.

Proof. Deny. Choose an atom $\alpha=\left(a_{n}\right)$ of $A$. As $\alpha \neq 0$, we can assume that $a_{n} \neq 0$ for each $n$ (by passing to some $\left(a_{n}\right)_{n \geq m}$ and using cofinality). By Theorem 2.5 (c), $a_{n}$ is a nonunit of $A_{n}$ for each $n$. By hypothesis, $a_{1}$ is not an atom of $A_{1}$, and so $a_{1}=b_{1} c_{1}$ for some $b_{1}, c_{1} \in A_{1} \backslash \mathscr{U}\left(A_{1}\right)$. Pick $b_{2}^{*} \in \varphi_{2,1}^{-1}\left(b_{1}\right)$ and $c_{2} \in \varphi_{2,1}^{-1}\left(c_{1}\right)$. Then $a_{2}-b_{2}^{*} c_{2} \in \operatorname{ker}\left(\varphi_{2,1}\right)=Q_{2,1}$. Now, $c_{2} \notin Q_{2,1}$ since $c_{1} \neq 0$. Since Proposition 2.4 (c) ensures that $Q_{2,1}$ is a divided prime ideal of 
$A_{2}$, we have $Q_{2,1} \subseteq A_{2} c_{2}$, and so $a_{2}-b_{2}^{*} c_{2}=c_{2} d_{2}$ for some $d_{2} \in A_{2}$. It follows that $a_{2}=b_{2} c_{2}$, with $b_{2}:=b_{2}^{*}+d_{2} \in A_{2}$. Since

$$
0=\varphi_{2,1}\left(c_{2} d_{2}\right)=\varphi_{2,1}\left(c_{2}\right) \varphi_{2,1}\left(d_{2}\right)=c_{1} \varphi_{2,1}\left(d_{2}\right)
$$

and $A_{1}$ is an integral domain, $\varphi_{2,1}\left(d_{2}\right)=0$. Therefore, $\varphi_{2,1}\left(b_{2}\right)=\varphi_{2,1}\left(b_{2}^{*}\right)+$ $\varphi_{2,1}\left(d_{2}\right)=b_{1}+0=b_{1}$. Iterating the above argument, we find for each $n \in \mathbf{N}$, nonunits $b_{n}$ and $c_{n}$ of $A_{n}$ such that $a_{n}=b_{n} c_{n}, \varphi_{n+1, n}\left(b_{n+1}\right)=b_{n}$, and $\varphi_{n+1, n}\left(c_{n+1}\right)=c_{n}$. Evidently, $\beta:=\left(b_{n}\right)$ and $\gamma:=\left(c_{n}\right)$ are in $A$; in fact, they are nonunits of $A$, by Theorem 2.5 (c). However, $\alpha=\beta \gamma$, contradicting that $\alpha$ is an atom of $A$.

REMARK 2.9. Following [3], an integral domain $D$ is an antimatter domain if $D$ has no atoms. As in [6], $D$ is a fragmented domain if, for each $d \in D \backslash \mathscr{U}(D)$, there exists $e \in D \backslash \mathscr{U}(D)$ such that $d \in \bigcap_{n=1}^{\infty} D e^{n}$. Any fragmented domain is an antimatter domain, but the converse is false. Indeed, for each $n \in \mathrm{N}$, [3, Corollary 3.11 (b)] uses repeated Nagata compositions to produce an $n$-dimensional antimatter valuation domain $A_{n}$ (therein denoted $V_{n}$ ); and $A_{n}$ is not fragmented since quasilocal fragmented domains are either fields or infinite-dimensional [6, Corollary 2.8]. What about $A:=\lim A_{n}$ ? We see quickly that $A$ is a valuation domain (by Theorem 2.1 (g)) $\overleftarrow{\operatorname{dim}}(A)=\infty$ (by Corollary 2.6), and $A$ is an antimatter domain (by Proposition 2.8). To answer the question left open in [3], we now show that $A$ is not fragmented. Since $A$ is a valuation domain, [6, Corollary 2.6] translates the problem to showing that $M$, the maximal ideal of $A$, is not unbranched in $A$; that is, that $M$ is not the union of the nonmaximal prime ideals of $A$. In view of Theorem 2.5 (a), (b), this conclusion follows from the analogous fact about $A_{2}$.

Theorems 2.10 and 2.12 address the integral closure of $A$.

Theorem 2.10. Assume that $K_{n}=q f\left(A_{n}\right)$ for each $n \in \mathrm{N}$. Then the following conditions are equivalent:

(1) For each $n \in \mathrm{N}, A_{n}$ is integrally closed;

(2) $A_{1}$ is integrally closed and, for each $n \in \mathrm{N}, B_{n}$ is integrally closed;

(3) $A$ is integrally closed.

Proof. If $P$ is a divided prime ideal of an integral domain $D$, we have the pullback description $D=D_{P} \times_{D_{P} / P} D / P$. It follows (cf. [11, Corollary 1.5 (5)]) that $D$ is integrally closed if and only if both $D_{P}$ and $D / P$ are integrally closed.

$(3) \Rightarrow(1)$ : Assume (3) and fix $n \in \mathrm{N}$. By Proposition 2.4 (d), $Q_{n}$ is a divided prime ideal of $A$. Hence, by the above remark, $A / Q_{n}$ is integrally closed. Then (1) follows since $A / Q_{n} \cong A_{n}$ by Lemma 2.2 (b). 
$(1) \Rightarrow(3)$ : It suffices to show that if $\xi \in A^{\prime}$, then $\xi \in A$. Write $\xi=a b^{-1}$, with $a \in A$ and $0 \neq b \in A$. By Lemma 2.2 (c), there exists $m \in \mathrm{N}$ such that $b \notin Q_{m}$. Since $\xi$ is integral over $A$, it is easy to see that $\eta:=\left(a+Q_{m}\right)\left(b+Q_{m}\right)^{-1} \in q f\left(A / Q_{m}\right)$ is integral over $A / Q_{m} \cong A_{m}$. Since $A_{m}$ is integrally closed by (1), $\eta=c+Q_{m}$ for some $c \in A$. It follows that $q:=a-b c \in Q_{m}$. As $Q_{m}$ is a divided prime ideal of $A$ (by Proposition 2.4 (d)) and $b \notin Q_{m}$, we have $Q_{m} \subseteq A b$. In particular, $q \in A b$, and so $q b^{-1} \in A$. Hence, $\xi=c+q b^{-1} \in A+A \subseteq A$.

(1) $\Leftrightarrow(2)$ : Since $K_{n}=q f\left(A_{n}\right)$, the pullback $A_{n+1}=B_{n} \times_{K_{n}} A_{n}$ leads canonically to $M_{n}=\operatorname{ker}\left(\varphi_{n}\right)=\operatorname{ker}\left(\varphi_{n+1, n}\right), B_{n}=\left(A_{n+1}\right)_{M_{n}}$, and $A_{n}=A_{n+1} / M_{n}$. Hence, by the first comment of the proof, $A_{n+1}$ is integrally closed if and only if both $B_{n}$ and $A_{n}$ are integrally closed. The assertion now follows by induction on $n$.

It is of some technical interest to note that the proof of the equivalence (1) $\Leftrightarrow(3)$ in Theorem 2.10 did not use the hypothesis that $K_{n}=q f\left(A_{n}\right)$ for each $n$. Also, the following condition can be added in those in Theorem 2.10:

(4) there exists $m \in \mathrm{N}$ such that $A_{n}$ is integrally closed for each $n \geq m$.

Lemma 2.11. Let $C_{1}$ be an overring of $A_{1}$ which is not a field. For $n \in \mathrm{N}$, define $C_{n+1}$ inductively by $C_{n+1}:=B_{n} \times_{K_{n}} C_{n}=\varphi_{n}^{-1}\left(C_{n}\right)$. If $m \geq n$ in $\mathrm{N}$, let $\varphi_{m, n}: C_{m} \rightarrow C_{n}$ denote the natural extension of $\varphi_{m, n}: A_{m} \rightarrow A_{n}$. Consider the inverse system generated by $\left\{C_{1} ;\left(K_{n}, B_{n}\right): n \in \mathrm{N}\right\}$, and put $C:=\lim _{\leftarrow} C_{n}$. Then:

(a) For each $n \in \mathrm{N}, M_{n}=\left(A_{n+1}: B_{n}\right)=\left(C_{n+1}: B_{n}\right)$.

(b) If $m>n$ in $\mathrm{N}$, then $Q_{m, n}=\operatorname{ker}\left(\varphi_{m, n}: A_{m} \rightarrow A_{n}\right)=\varphi_{m, n+1}^{-1}\left(M_{n}\right)=$ $\operatorname{ker}\left(\varphi_{m, n}: C_{m} \rightarrow C_{n}\right)$.

(c) For each $n \in \mathrm{N}, Q_{n}$ is a divided prime ideal of $C$.

(d) $A$ and $C$ have the same quotient field and the same complete integral closure.

Proof. The assertions in (a) and (b) follow easily from Proposition 2.4 (a) since $M_{n} \neq 0$. Moreover, replacing $A_{1}$ with $C_{1}$ in the earlier discussion, we see that (c) follows from (b) and Proposition 2.4 (b), (d). Finally, since Proposition 2.4 (a) ensures that $Q_{1} \neq 0$, (d) follows since $A$ and $C$ share a common nonzero ideal (cf. [15, Theorem 13.1 (3)]).

THEOREM 2.12. Assume that for each $n \in \mathrm{N}, B_{n}$ is integrally closed and $K_{n}=q f\left(A_{n}\right)$. Then $A^{\prime}$ is the inverse limit of the integral closures $A_{n}^{\prime}$ of $A_{n}$; that is, $\left(\lim _{\leftarrow} A_{n}\right)^{\prime}=\lim _{\leftarrow} A_{n}^{\prime}$.

Proof. Since $B_{n}^{\prime}=B_{n}$ by hypothesis, it follows that $A_{n+1}^{\prime} \subseteq B_{n}$ and (cf. [11, Corollary $1.5(5)]) A_{n+1}^{\prime}=B_{n} \times_{K_{n}} A_{n}^{\prime}$. As in Lemma 2.11, consider the inverse system generated by $\left\{A_{1}^{\prime} ;\left(K_{n}, B_{n}\right): n \in \mathrm{N}\right\}$. (The earlier theory ap- 
plies since, by integrality, $A_{1}^{\prime}$ inherits from $A_{1}$ the property of not being a field.) Put $R:=\lim A_{n}^{\prime}$. By Theorem $2.10, R$ is integrally closed; and by Lemma 2.11 (d), $\overleftarrow{A}$ and $R$ have the same quotient field. As lim preserves inclusions, $A=\lim _{\leftarrow} A_{n} \subseteq \lim _{\leftarrow} A_{n}^{\prime}=R$, and so it suffices to prove that $R$ is integral over $A$.

Let $x=\left(x_{n}\right) \in R \subseteq \prod A_{n}^{\prime}$. Since $x_{1} \in A_{1}^{\prime}$, we have

$$
x_{1}^{r}+a_{r-1}^{(1)} x_{1}^{r-1}+\cdots+a_{1}^{(1)} x_{1}+a_{0}^{(1)}=0
$$

for some $r \in \mathrm{N}$ and elements $a_{k}^{(1)} \in A_{1}$. For each $n \in \mathrm{N}$, if $0 \leq k \leq r-1$, choose $b_{k}^{(n)} \in \varphi_{n, 1}^{-1}\left(a_{k}^{(1)}\right) \in A_{n}$; of course, $b_{k}^{(1)}=a_{k}^{(1)}$. For $n=2$, put

$$
y_{2}:=x_{2}^{r}+b_{r-1}^{(2)} x_{2}^{r-1}+\cdots+b_{1}^{(2)} x_{2}+b_{0}^{(2)} \in A_{2}^{\prime} .
$$

As $\varphi_{2,1}$ is a homomorphism and $\varphi_{2,1}\left(x_{2}\right)=x_{1}$, we have $\varphi_{2,1}\left(y_{2}\right)=0$. Hence

$$
c_{0}^{(2)}:=-y_{2} \in \operatorname{ker}\left(\varphi_{2,1}\right) \subseteq A_{2}
$$

satisfies $x_{2}^{r}+b_{r-1}^{(2)} x_{2}^{r-1}+\cdots+b_{1}^{(2)} x_{2}+\left(b_{0}^{(2)}+c_{0}^{(2)}\right)=0$.

Since $c_{0}^{(2)} \in A_{2}$, we have $a_{0}^{(2)}:=b_{0}^{(2)}+c_{0}^{(2)} \in A_{2}$. Also, if $1 \leq k \leq r-1$, then $a_{k}^{(2)}:=b_{k}^{(2)} \in A_{2}$. Repeating the above argument, we find elements $a_{k}^{(3)} \in A_{3}$, $0 \leq k \leq r-1$, such that

$$
x_{3}^{r}+a_{r-1}^{(3)} x_{3}^{r-1}+\cdots+a_{1}^{(3)} x_{3}+a_{0}^{(3)}=0
$$

and $\varphi_{3,2}\left(a_{k}^{(3)}\right)=a_{k}^{(2)}$ for all $k$. Iterating the argument, we thus produce $a_{k}=\left(a_{k}^{(n)}\right) \in A, 0 \leq k \leq r-1$, such that for each $n \in \mathrm{N}$,

$$
x_{n}^{r}+a_{r-1}^{(n)} x_{n}^{r-1}+\cdots+a_{1}^{(n)} x_{n}+a_{0}^{(n)}=0 .
$$

Therefore, $x^{r}+a_{r-1} x^{r-1}+\cdots+a_{1} x+a_{0}=0$; that is, $x$ is integral over $A$.

In explaining that $A_{1}^{\prime}$ is not a field, the proof of Theorem 2.12 appealed to the lying-over theorem (cf. [19, Theorem 44]). It is natural to ask if LO (as well as GU, GD and INC) satisfies an analogue of Theorem 2.12; in particular, if (the above type of) lim preserves LO. Our next result gives an affirmative answer. It is convenient to consider also the following property which was introduced in [21]. Recall that an inclusion $f: D \rightarrow E$ of integral domains is an i-extension if $\operatorname{Spec}(f): \operatorname{Spec}(E) \rightarrow \operatorname{Spec}(D)$ is an injection.

Proposition 2.13. As above, consider the inverse system generated by $\left\{A_{1} ;\left(K_{n}, B_{n}\right): n \in \mathrm{N}\right\}$, with $A=\lim A_{n}$. Consider a(nother) inverse system generated by $\left\{A_{* 1} ;\left(K_{* n}, B_{* n}\right): n \in \overleftarrow{\mathrm{N}}\right\}$, with $A_{*}=\lim A_{* n}$; in the latter system, denote structures $\varphi_{* m, n}, \Phi_{* n}, Q_{* r, n}, Q_{* n}$ analogously to the corresponding "unstarred" structures in the former system. For each $n \in \mathrm{N}$, suppose given an injective ring-homomorphism $h_{n}: A_{n} \rightarrow A_{* n}$; and suppose that $\varphi_{*(n+1), n} \circ h_{n+1}=$ 
$h_{n} \circ \varphi_{n+1, n}$ for each $n \in \mathrm{N}$. Let $h: A \rightarrow A_{*}$ be the induced injective ring-homomorphism. Then:

(a) Let $Q \in \operatorname{Spec}\left(A_{*}\right)$ and $P:=h^{-1}(Q)=Q \cap A \in \operatorname{Spec}(A)$. Then $Q=0$ if and only if $P=0$.

(b) Fix $n \in$ N. Consider $Q \in \operatorname{Spec}\left(A_{* n}\right) \subseteq \operatorname{Spec}\left(A_{*}\right)$ and $P \in \operatorname{Spec}\left(A_{n}\right) \subseteq$ $\operatorname{Spec}(A)$. Then $h_{n}^{-1}(Q)=P$ if and only if $h^{-1}(Q)=P$.

(c) Let $\mathscr{P}$ be one of the following five properties: $L O, G U, G D, I N C$, "(is an) i-extension". If $h_{n}$ satisfies $\mathscr{P}$ for each $n \in \mathrm{N}$, then $h$ satisfies $\mathscr{P}$.

Proof. (a) It is useful to view $h$ and the maps $h_{n}$ as inclusions occasionally, and the notation " $Q \cap A$ " in the statement of (a) is interpreted in this sense. (Of course, $h$ is an injection since lim preserves injections.) Now, of course, $0 \cap A=0$. Thus, it suffices to show that if $Q \neq 0$, then $P \neq 0$. By Theorem 2.5 (a), we can view $Q \in \operatorname{Spec}\left(A_{* n}\right)$ for some $n \in \mathrm{N}$. Since $\Phi_{* n} \circ h=h_{n} \circ \Phi_{n}: A \rightarrow A_{* n}$, functoriality of Spec gives a commutative diagram

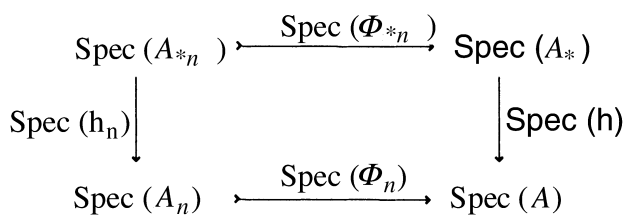

whose horizontal maps are viewed as inclusions. Chasing $Q$ through this diagram, we find that $P=h^{-1}(Q)$ is (the canonical image of) $h_{n}^{-1}(Q) \in \operatorname{Spec}\left(A_{n}\right)$. By Theorem 2.5 (a), $h_{n}^{-1}(Q) \neq 0 \in \operatorname{Spec}(A)$, and so $P \neq 0$.

(b) This follows from the above commutative-diagram argument.

(c) All five proofs are similar. We give the proof for $\mathscr{P}=\mathrm{GU}$ and leave the other proofs to the reader. Thus, we suppose given primes $P_{1} \subseteq P_{2}$ in $\operatorname{Spec}(A)$ and $P_{* 1} \in \operatorname{Spec}\left(A_{*}\right)$ such that $h^{-1}\left(P_{* 1}\right)=P_{1}$, and we seek a prime $P_{* 2} \in \operatorname{Spec}\left(A_{*}\right)$ such that $h^{-1}\left(P_{* 2}\right)=P_{2}$ and $P_{* 1} \subseteq P_{* 2}$. Without loss of generality, $P_{1} \neq P_{2}$.

Case 1: $P_{1}=0$. By (a), $P_{* 1}=0$. It suffices to find $P_{* 2} \in \operatorname{Spec}\left(A_{*}\right)$ such that $h^{-1}\left(P_{* 2}\right)=P_{2}$. Using Theorem 2.5 (a), choose $n \in \mathrm{N}$ such that $P_{2} \in \operatorname{Spec}\left(A_{n}\right)$. Since $h_{n}$ is an injection which satisfies GU, [19, Theorem 42] ensures that $h_{n}$ satisfies LO, and so there exists $P_{* 2} \in \operatorname{Spec}\left(A_{* n}\right)$ such that $h_{n}^{-1}\left(P_{* 2}\right)=P_{2}$. Then, by (b), $h^{-1}\left(P_{* 2}\right)=P_{2}$.

Case 2: $P_{1} \neq 0$. By (a), $P_{* 1} \neq 0$. By applying Theorem 2.5 (a) three times and choosing the maximal of three subscripts, we find $n \in \mathrm{N}$ such that $P_{1}, P_{2} \in \operatorname{Spec}\left(A_{n}\right)$ and $P_{* 1} \in \operatorname{Spec}\left(A_{* n}\right)$. Next, note that the injections $\operatorname{Spec}\left(\Phi_{n}\right)$ and $\operatorname{Spec}\left(\Phi_{* n}\right)$ both preserve and reflect order (that is, inclusions 
of prime ideals). Thus, $P_{1} \subseteq P_{2}$ when viewed in $\operatorname{Spec}\left(A_{n}\right)$, and so, in view of (b), it suffices to find $P_{* 2}$ in $\operatorname{Spec}\left(A_{n}\right)$ such that $P_{* 1} \subseteq P_{* 2}$ when viewed in $\operatorname{Spec}\left(A_{* n}\right)$ and $h_{n}^{-1}\left(P_{* 2}\right)=P_{2}$. This, in turn, is accomplished since $h_{n}$ satisfies GU.

Recall from [21] that an integral domain $D$ is an $i$-domain in case the inclusion map $D \rightarrow E$ is an $i$-extension for each overring $E$ of $D$. Evidently, being an $i$-domain is a local property of integral domains. It was shown in [21, Proposition 2.14 and Corollary 2.15] that an integral domain $D$ is an $i$ domain if and only if $D \subseteq D^{\prime}$ is an $i$-extension and $D^{\prime}$ is a Prüfer domain; and that an integral domain $D$ is a quasilocal $i$-domain if and only if $D^{\prime}$ is a valuation domain.

Proposition 2.14. Assume that for each $n \in \mathrm{N}, B_{n}$ is integrally closed and $K_{n}=q f\left(A_{n}\right)$. Then the following conditions are equivalent:

(1) For each $n \in \mathrm{N}, A_{n}$ is a quasilocal $i$-domain;

(2) $A_{1}$ is a quasilocal $i$-domain and, for each $n \in \mathrm{N}, B_{n}$ is a valuation domain;

(3) $A$ is a quasilocal i-domain.

Proof. By Theorem 2.5 (b), $A$ is quasilocal $\Leftrightarrow A_{n}$ is quasilocal for each $n \Leftrightarrow A_{1}$ is quasilocal. Thus, we may assume henceforth that each $A_{n}$ is quasilocal. By Lemma 2.11 and Theorem 2.12, we have $A^{\prime}=\lim A_{n}^{\prime}$, with $A_{n}^{\prime}=A^{\prime} / Q_{n}$ for each $n \in \mathrm{N}$ (cf. [11, Corollary 1.5 (5)]). In particular, if $A^{\prime}$ is a valuation domain, then so is each $A_{n}^{\prime}$. Thus, in view of the material recalled from [21], $(3) \Rightarrow(1)$. On the other hand, $(1) \Rightarrow(3)$ by Theorem 2.12 and Theorem $2.1(\mathrm{~g})$, for (1) leads to $A^{\prime}$ being an inverse limit of valuation domains. By Corollary 2.7, if $A_{n}^{\prime}$ is a valuation domain for each $n$, then $B_{n}$ is a valuation domain for each $n$. Consequently, $(1) \Rightarrow(2)$. Finally, to show that $(2) \Rightarrow(1)$, assume (2), observe that $A_{1}^{\prime}$ is a valuation domain, and use Corollary 2.7 to conclude that $A^{\prime}$ is a valuation domain.

The above material leads one naturally to ask if (the ambient type of) lim preserves Prüfer domains or (not necessarily quasilocal) $i$-domains. For this reason, we next address localizations of inverse limits. As usual, if $D$ is an integral domain and $P \in \operatorname{Spec}(D)$, it is convenient to let $\mathrm{k}_{D}(P)$ denote $q f(D / P)=D_{P} / P D_{P}$.

Proposition 2.15. Let $0 \neq P \in \operatorname{Spec}(A)$. Choose $m \in \mathrm{N}$ such that $P \in \operatorname{Spec}\left(A_{m}\right)$; thus, $P \supseteq Q_{m}$. (Such $m$ exists by Theorem $2.5(a)$.) Then for each $n \geq m$ in $\mathrm{N}, P_{n}:=\Phi_{n}(P)$ is a prime ideal of $A_{n}$. Then:

(a) $P \cong \lim \left\{P_{n}: n \geq m\right\}$.

(b) $A / P \cong A_{n} / P_{n}$ for each $n \geq m$, and so $A / P \cong \lim \left\{A_{n} / P_{n}: n \geq m\right\}$. 
(c) $\mathrm{k}_{A}(P) \cong \mathrm{k}_{A_{n}}\left(P_{n}\right)$ for each $n \geq m$, and so $\mathrm{k}_{A}(P) \cong \lim \left\{\mathrm{k}_{A_{n}}\left(P_{n}\right): n \geq m\right\}$.

(d) $A_{P} \cong \lim \left\{\left(A_{n}\right)_{P_{n}}: n \geq m\right\}$, the inverse limit of the inverse system generated by $\left\{\left(A_{m}\right)_{P_{m}} ;\left(K_{n}, B_{n}\right): n \geq m\right\}$.

Proof. (a) Observe that $P_{m}=P$. Using Theorem 2.1 (d) and Lemma 2.2 (b), notice that $\varphi_{r, s}^{-1}\left(P_{s}\right)=P_{r}$ if $r \geq s \geq m$. It follows that $P \hookrightarrow A=$ $\lim \left\{A_{n}: n \geq m\right\}$ factors through $\lim \left\{P_{n}: n \geq m\right\}$, and (a) follows.

(b), (c): Notice that $P_{n} \in \operatorname{Spec}(\overleftarrow{A})$ is identified with $P / Q_{n} \in \operatorname{Spec}\left(A / Q_{n}\right)=$ $\operatorname{Spec}\left(A_{n}\right)$ for each $n \geq m$. Then $A / P \cong A_{n} / P_{n}$ by a standard isomorphism theorem. These isomorphisms are compatible with the isomorphisms $A_{n+1} / P_{n+1} \stackrel{\cong}{\longrightarrow} A_{n} / P_{n}$ induced by $\varphi_{n+1, n}$, and (b), (c) follow easily.

(d) If $n \geq m$, we identify $\Phi_{n+2}(P)=P \supseteq Q_{m} \supseteq Q_{n+1}$, and so Proposition 2.4 (a) leads to

$$
P \supseteq \Phi_{n+2}\left(Q_{n+1}\right)=Q_{n+2, n+1}=M_{n} .
$$

Thus, by the universal mapping property of localizations, the inclusion map $A_{n+1} \rightarrow B_{n}$ extends to an inclusion $\left(A_{n+1}\right)_{P_{n+1}} \rightarrow B_{n}$. Moreover, since $\varphi_{n+1, n}\left(A_{n+1} \backslash P_{n+1}\right)=A_{n} \backslash P_{n}$, the surjection $\varphi_{n+1, n}$ induces a surjection $\left(A_{n+1}\right)_{P_{n+1}} \rightarrow\left(A_{n}\right)_{P_{n}}$. In particular, $\varphi_{n}^{-1}\left(\left(A_{n}\right)_{P_{n}}\right)=\left(A_{n+1}\right)_{P_{n+1}}$ and we have a pullback description $\left(A_{n+1}\right)_{P_{n+1}}=B_{n} \times_{K_{n}}\left(A_{n}\right)_{P_{n}}$. Let $m$ be increased, if necessary, so that $P$ properly contains $Q_{m}$. Then we can let $R:=\lim _{\leftarrow}\left(A_{n}\right)_{P_{n}}$, the inverse limit of the inverse system generated by $\left\{\left(A_{m}\right)_{P_{m}} ;\left(K_{n}, B_{n}\right): n \geq m\right\}$. By Lemma 2.11 (d), $R$ has he same quotient field, say $K$, as $A$ (and, hence, the same as $A_{P}$ ). Moreover, the universal mapping property of localization gives ring-homomorphisms $A_{P} \rightarrow\left(A_{n}\right)_{P_{n}}$ which, in view of the universal mapping property of $\lim _{\leftarrow}$, lead to a ring-homomorphism $A_{P} \rightarrow R$. As this map is evidently injective, we view it as an inclusion. It remains only to prove that $A_{P}=R$. To this end, observe via (a) that

$$
\begin{aligned}
A_{P}= & \left\{a b^{-1} \in K: a \in A, b \in A \backslash P\right\}=\left\{\left(a_{n}: n \geq 1\right)\left(b_{n}: n \geq 1\right)^{-1} \in K:\right. \\
& \left.a=\left(a_{n}\right) \in A, \quad b=\left(b_{n}\right) \in A, \quad b_{n} \in A_{n} \backslash P_{n} \text { for each } n \geq m(b) \geq m \geq 1\right\} \\
& \supseteq \lim _{\leftarrow}\left\{\left(A_{n}\right)_{P_{n}}: n \geq m\right\}=R .
\end{aligned}
$$

Corollary 2.16. Let $0 \neq P \in \operatorname{Spec}(A)$. As in Proposition 2.15, choose $m \in \mathrm{N}$ such that $P \in \operatorname{Spec}\left(A_{m}\right)$ properly contains $Q_{m}$, and put $P_{n}:=\Phi_{n}(P)$ for each $n \geq m$ in $\mathrm{N}$. Then $P$ is a divided prime ideal of $A$ if and only if $P_{n}$ is a divided prime ideal of $A_{n}$ for each (resp., some) $n \geq m$.

Proof. If $Q$ is a divided prime ideal of an integral domain $D$ and $Q$ contains $I \in \operatorname{Spec}(D)$, then $Q / I$ is a divided prime ideal of $D / I$ (cf. [5, Lemma 2.2 (c)]). Thus, if $P$ is divided in $A$ and $n \geq m$ in $\mathrm{N}$, then $P_{n} \cong P / Q_{n}$ is divided in $A / Q_{n} \cong A_{n}$. For the converse, suppose that $P_{m}$ is divided in $A_{m}$; that 
is, $A_{m}=\left(A_{m}\right)_{P_{m}} \times_{\mathrm{k}_{A_{m}}\left(P_{m}\right)} A_{m} / P_{m}$. Now, by the proof of Proposition $2.15(\mathrm{~d})$, $\left(A_{n+1}\right)_{P_{n+1}}=B_{n} \times_{K_{n}}\left(A_{n}\right)_{P_{n}}$ for each $n \geq m$. It follows from the pullback description of $A_{n}$ that for each $n \geq m$, we have a canonical pullback diagram

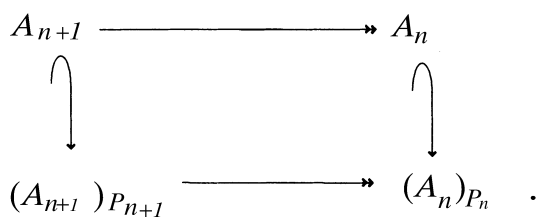

In cases like this, lim commutes with pullbacks [18, Theorem 5.2, page 277]. Thus, by Proposition 2.15 (d), we have, with $n \geq m$, a pullback diagram

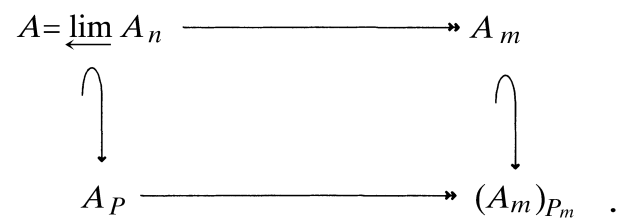

Since $P_{m}$ is divided in $A_{m}$, Proposition 2.15 (b), (c) gives a pullback diagram

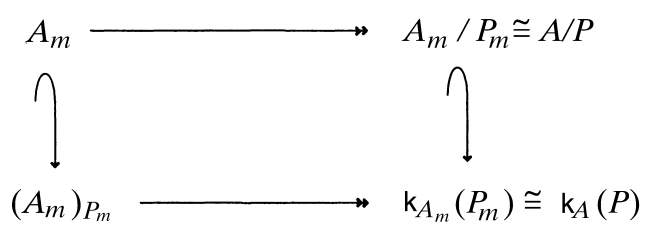

Juxtaposing the last two pullback diagrams, we conclude that $A=A_{P} \times_{\mathrm{k}_{A}(P)} A / P$; that is, $P$ is divided in $A$.

In Corollary 2.16, it may be shown directly, using the dividedness of $Q_{n, m}$ (Proposition 2.4 (c)), that if $P_{m}$ is divided, then so is $P_{n}$ for each $n \geq m$.

COROLlary 2.17. (a) The following two conditions are equivalent:

(1) For each $n \in \mathrm{N}, A_{n}$ is a divided domain;

(2) $A$ is a divided domain.

Moreover, if $K_{n}=q f\left(A_{n}\right)$ for each $n$, then (1) and (2) are each equivalent to

(3) $A_{1}$ is a divided domain and, for each $n \in \mathrm{N}, B_{n}$ is a divided domain.

(b) The assertions in (a) are valid if "divided" is replaced throughout by "locally divided".

(c) Suppose that for all $n \in \mathrm{N}, B_{n}$ is integrally closed and $K_{n}=q f\left(A_{n}\right)$. Then the following conditions are equivalent: 
(1) For each $n \in \mathrm{N}, A_{n}$ is an i-domain;

(2) $A$ is an i-domain;

(3) $A_{1}$ is an i-domain and, for each $n \in \mathrm{N}, B_{n}$ is a valuation domain.

Proof. (a) Since each $A_{n}$ is (isomorphic to) a factor domain of $A$, $(2) \Rightarrow(1)$ by $[5$, Lemma 2.2 (c)]. Since the zero prime ideal is divided in any integral domain, $(1) \Rightarrow(2)$ follows from the "if" assertion in Corollary 2.16. Next, suppose that $K_{n}=q f\left(A_{n}\right)$ for each $n \in \mathrm{N}$. Since $A_{n+1}=B_{n} \times_{K_{n}} A_{n}$, it can be shown that $A_{n+1}$ is a divided domain if and only if both $B_{n}$ and $A_{n}$ are divided domains. (This assertion is essentially a translation of the first assertion in [7, Proposition 2.12].) It is now evident that (3) $\Leftrightarrow(1)$.

(b) Any factor domain of a locally divided domain is locally divided [5]. Hence, $(1) \Leftrightarrow(2)$ by (a) and Proposition 2.15 (d). For a proof that $(3) \Leftrightarrow(1)$, assuming that $K_{n}=q f\left(A_{n}\right)$ for each $n$, use the following translation of the second assertion in [7, Proposition 2.12]: $A_{n+1}$ is locally divided if and only if both $B_{n}$ and $A_{n}$ are locally divided.

(c) $(2) \Rightarrow(1)$ since $D / P$ is an $i$-domain whenever $P$ is a (divided) prime ideal of an $i$-domain $D[21$, p. 3]. Next, since being an $i$-domain is a local property, $(1) \Rightarrow(2)$ follows by combining Propositions 2.15 and 2.14. A similar combination yields $(1) \Leftrightarrow(3)$, once we notice that $A$ has a nonzero prime ideal (cf. Theorem 2.5 (b)).

The next result will be used in determining when $A$ is a $P^{r} V D$.

Corollary 2.18. Fix $n \in \mathrm{N}$, and suppose that $K_{m}=q f\left(A_{m}\right)$ for each $m \geq n$ in $\mathrm{N}$. For $m \geq n+1$, inductively define $\left(B_{n}^{\bullet}\right)_{m}$ by

$$
\begin{aligned}
& \left(B_{n}^{\bullet}\right)_{n+1}:=\varphi_{n+1}^{-1}\left(B_{n}\right) \subseteq B_{n+1}, \\
& \left(B_{n}^{\bullet}\right)_{m+1}:=\varphi_{m+1}^{-1}\left(\left(B_{n}^{\bullet}\right)_{m}\right) \subseteq B_{m+1} \text { if } m \geq n+1 .
\end{aligned}
$$

Consider the inverse system generated by $\left\{\left(B_{n}^{\bullet}\right)_{n+1} ;\left(K_{m}, B_{m}\right): m \geq n+1\right\}$, and put $B_{n}^{\bullet}=\lim _{\leftarrow}\left\{\left(B_{n}^{\bullet}\right)_{m}: m \geq n+1\right\}$. Then $B_{n}^{\bullet}=A_{Q_{n}}$.

Proof. The inverse system in question satisfies our riding hypotheses. (The only issue may concern whether $\left(B_{n}^{\bullet}\right)_{n+1}$ is a field; if so, formally introduce $\left(B_{n}^{\bullet}\right)_{n}:=B_{n}$, which is not a field.) Now, by Proposition 2.4 (a), $\Phi_{n+1}\left(Q_{n}\right)=M_{n}$; and, since $K_{n}=q f\left(A_{n}\right),\left(A_{n+1}\right)_{\Phi_{n+1}\left(Q_{n}\right)}=\left(B_{n}^{\bullet}\right)_{n+1}$ (cf. also [14, Lemma 1.1.6]). Similarly, one shows by induction on $m$ that $\left(A_{m}\right)_{\Phi_{m}\left(Q_{n}\right)}=\left(B_{n}^{\bullet}\right)_{m}$ for each $m \geq n+1$. Now, since Proposition 2.4 (a) ensures that $Q_{n} \neq 0$, Proposition 2.15 (d) may be applied to $P:=Q_{n}$ and the above inverse system, with the result that

$$
B_{n}^{\bullet}=\lim _{\leftarrow}\left(B_{n}^{\bullet}\right)_{m}=\lim _{\leftarrow}\left(A_{m}\right)_{\Phi_{m}\left(Q_{n}\right)}=A_{Q_{n}} .
$$


Recall from [17] that an integral domain $D$ is a pseudo-valuation domain (PVD) if $D$ has a valuation overring $V$ such that $\operatorname{Spec}(D)=\operatorname{Spec}(V)$ as sets. Any $P V D$ is quasilocal and, in fact, a divided domain. It is useful to recall from [17, Proposition 2.6] that if $D$ is a $P V D$ and $P \in \operatorname{Spec}(D)$ is nonmaximal, then $D_{P}$ is a valuation domain; and from [2, Proposition 2.6] that $D$ is a $P V D$ if and only if $D=V \times_{k} F$, where $V$ is a valuation domain with residue field $k$ and $F$ is a subfield of $k$.

From [12, Définition 1.2 and Théorème 1.3] recall that a pseudo-valuation domain of type $r\left(P^{r} V D\right)$ can be defined, by induction on $r \geq 0$, in the following way. A $P^{0} V D$ is a $P V D$ and, for $r \geq 1$, a $P^{r} V D, D$, is defined by a pullback diagram of the following type:

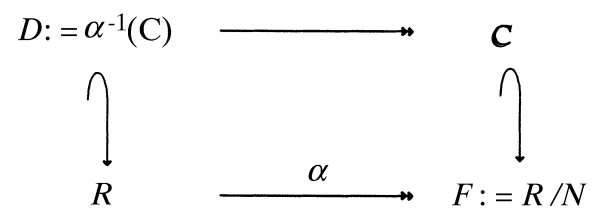

where $R$ is a $P^{r-1} V D$, with maximal ideal $N, F$ is its residue field, $\alpha$ is the canonical projection and $C$ is a $P V D$ with field of quotients isomorphic to $F$. For instance if $k$ is a field and $r \geq 0$, then

$$
\begin{aligned}
k & +X_{1} k\left(\left(Y_{1}\right)\right)\left[\left[X_{1}\right]\right]+X_{2} k\left(\left(X_{1}, Y_{1}\right)\right)\left[\left[X_{2}\right]\right]+\cdots \\
& +X_{r+1} k\left(\left(X_{1}, Y_{1}, \ldots, X_{r}, Y_{r}\right)\right)\left[\left[X_{r+1}\right]\right]
\end{aligned}
$$

is a $P^{r} V D$.

Proposition 2.19. Let $0 \leq r \in \mathrm{Z}$. Then the following conditions are equivalent:

(1) There exists $m \in \mathrm{N}$ such that, for each $n \geq m$ in $\mathrm{N}, A_{n}$ is a $P^{r} V D$;

(2) There exists $m \in \mathrm{N}$ such that $A_{m}$ is a $P^{r} V D$ and, for each $n \geq m$ in $\mathrm{N}, B_{n}$ is a valuation domain and $K_{n}=q f\left(A_{n}\right)$;

(3) $A$ is a $P^{r} V D$.

Proof. (3) $\Leftrightarrow(2)$ : By Proposition $2.4(\mathrm{~d}),\left\{Q_{n}: n \geq 1\right\}$ is a family of divided prime ideals of $A$. Using the definition of a $P^{r} V D$ (cf. also [12, Théorème 1.3]), we see that $A$ is a $P^{r} V D$ if and only if there exists $m \in \mathrm{N}$ such that $A / Q_{m}$ is a $P^{r} V D$ and $A_{Q_{m}}$ is a valuation domain. Of course, $A / Q_{m} \cong A_{m}$. Now, if $K_{n}=q f\left(A_{n}\right)$ for each $n \geq m$, Corollary 2.18 gives that

$$
A_{Q_{m}}=\lim _{\leftarrow}\left\{\left(B_{m}^{\bullet}\right)_{k}: k \geq m+1\right\}=B_{m}^{\bullet} .
$$

Therefore, applying Corollary 2.7 to the construction in Corollary 2.18, we 
see that $A_{Q_{m}}$ is a valuation domain if and only if, for each $k \geq m, B_{k}$ is a valuation domain and $K_{k+1}=q f\left(B_{k}\right)$. Thus, $(2) \Rightarrow(3)$. Moreover, by [12, Théorème 1.3] (3) implies that there exists $m \in \mathrm{N}$ such that $A_{m}$ is a $P^{r} V D$ and, for each $n \geq m$, that $K_{n}=q f\left(A_{n}\right)$ and so $(3) \Rightarrow(2)$.

(2) $\Leftrightarrow(1)$ : Given $K_{n}=q f\left(A_{n}\right)$, we see from the pullback $A_{n+1}=B_{n} \times_{K_{n}} A_{n}$ that $A_{n+1}$ is a $P^{r} V D$ if and only if $A_{n}$ is a $P^{r} V D$ and $B_{n}$ is a valuation domain (cf. [12, Théorème 1.3]). Thus, $(2) \Rightarrow(1)$. Moreover, in view of the above comments, $(1) \Rightarrow(2)$.

The next result is the $P V D$-theoretic analogue of Corollary 2.7 or of Proposition 2.19.

COROLlary 2.20. The following conditions are equivalent:

(1) For each $n \in \mathrm{N}, A_{n}$ is a PVD;

(2) $A_{1}$ is a PVD and, for each $n \in \mathrm{N}, B_{n}$ is a valuation domain and $K_{n}=q f\left(A_{n}\right)$;

(3) $A$ is a PVD.

Proof. It is well known that any factor domain of a $P V D$ is a $P V D$. One may use this fact, together with the material recalled above, to fashion a proof of Corollary 2.20. Alternatively, the first sentence of this proof may be combined with the case $r=0$ of the proof of Proposition 2.19; the upshot is another proof of Corollary 2.20, since a $P^{0} V D$ is the same as a $P V D$.

We next present the promised "globalization" of Corollary 2.7. Its proof depends on Corollary 2.7 in much the same way that Corollary 2.17 (b) was proved using Corollary 2.17 (a).

THEOREM 2.21. The following conditions are equivalent:

(1) For each $n \in \mathrm{N}, A_{n}$ is a Prüfer domain;

(2) $A_{1}$ is a Prüfer domain and, for each $n \in \mathrm{N}, B_{n}$ is a valuation domain and $K_{n}=q f\left(A_{n}\right)$;

(3) $A$ is a Prüfer domain.

Proof. (3) $\Rightarrow(1)$ since $A_{n} \cong A / Q_{n}$ and factor domains of Prüfer domains are Prüfer domains [15, Proposition 22.5]. For $(1) \Rightarrow(3)$, assume (1); it suffices to show that $A_{P}$ is a valuation domain for each $0 \neq P \in \operatorname{Spec}(A)$. By Proposition 2.15 (d), $A_{P}=\lim \left(A_{n}\right)_{P_{n}}$ for some $P_{n} \in \operatorname{Spec}\left(A_{n}\right)$, considering all $n \geq m=m(P)$. By (1), each $\left(A_{n}\right)_{P_{n}}$ is a valuation domain and so, by Theorem 2.1 (c), $A_{P}$ is also a valuation domain.

Since $A_{n+1}=B_{n} \times K_{n} A_{n}$, it follows from well-known material on pullbacks (cf. [11, Theorem 2.4 (3)]) that $A_{n+1}$ is a Prüfer domain if and only if $A_{n}$ is a Prüfer domain, $B_{n}$ is a (quasilocal Prüfer, that is) valuation domain, and $K_{n}=q f\left(A_{n}\right)$. Accordingly, $(2) \Leftrightarrow(1)$. 
Prüfer domains are the integrally closed $i$-domains (cf. [15]). In view of Theorem 2.21, Corollary 2.17 (c) and Proposition 2.13 (c), we are led to consider integral domains $D$ such that the inclusion map $D \rightarrow E$ satisfies INC for each overring $E$ of $D$. According to [21, Proposition 2.26], these are the integral domains $D$ for which $D^{\prime}$ is a Prüfer domain. These integral domains $D$ were called quasi-Prüfer domains by Ayache, Cahen and Echi; they have been studied extensively in [14].

Corollary 2.22. Suppose that for all $n \in \mathrm{N}, B_{n}$ is integrally closed and $K_{n}=q f\left(A_{n}\right)$. Then the following conditions are equivalent:

(1) For each $n \in \mathrm{N}, A_{n}$ is a quasi-Prüfer domain;

(2) $A_{1}$ is a quasi-Prüfer domain and, for each $n \in \mathrm{N}, B_{n}$ is a valuation domain;

(3) A is a quasi-Prüfer domain.

Proof. (3) $\Rightarrow(1)$ since $A_{n} \cong A / Q_{n}$ and $D / P$ is a quasi-Prüfer domain whenever $P$ is a (divided) prime ideal of a quasi-Prüfer domain $D$ [14, Proposition 6.5.1]. For $(1) \Rightarrow(3)$, combine Theorems 2.12 and $2.21[(1) \Rightarrow(3)]$. Finally, $(2) \Rightarrow(1)$ follows from the result that (if $B_{n}$ is integrally closed and quasilocal, then) $A_{n+1}=B_{n} \times_{K_{n}} A_{n}$ is a quasi-Prüfer domain if and only if $A_{n}$ is a quasi-Prüfer domain, $B_{n}$ is a valuation domain, and $K_{n}=q f\left(A_{n}\right)$ [14, Corollary 1.1.9 (1)].

We next consider when $A$ is a Bézout domain. Recall that an integral domain $D$ is a Bézout domain if each nonzero finitely generated ideal of $D$ is principal. Each Bézout domain is a Prüfer domain, but the converse is false (cf. [15]).

COROLlary 2.23. The following conditions are equivalent:

(1) For each $n \in \mathrm{N}, A_{n}$ is a Bézout domain;

(2) $A_{1}$ is a Bézout domain and, for each $n \in \mathrm{N}, B_{n}$ is a valuation domain and $K_{n}=q f\left(A_{n}\right)$;

(3) A is a Bézout domain.

Proof. It follows from [13, Theorem 4.2 (c)] that if $B^{*}$ is a quasilocal integral domain with residue field $K^{*}$ and $A^{*}$ is a (proper) subring of $K^{*}$, then $A^{* *}:=B^{*} \times_{K^{*}} A^{*}$ is a Bézout domain if and only if $A^{*}$ is a Bézout domain, $B^{*}$ is a valuation domain, and $K^{*}=q f\left(A^{*}\right)$. Thus, since $A_{n+1}=B_{n} \times_{K_{n}} A_{n}$, we see that $A_{n+1}$ is a Bézout domain if and only if $A_{n}$ is a Bézout domain, $B_{n}$ is a valuation domain, and $K_{n}=q f\left(A_{n}\right)$. Therefore, (2) $\Rightarrow(1)$. Moreover, since any factor domain of a Bézout domain is a Bézout domain [15], we also have that $(1) \Rightarrow(2)$ and (in light of Theorem $2.21[(3) \Rightarrow(2)])$ that $(3) \Rightarrow(2)$. It 
remains to show that [(1) and (2)] imply (3). Henceforth, assume (1) and (2); in particular, $K_{n}=q f\left(A_{n}\right)$ for each $n$.

Since $A_{1} \cong A / Q_{1}$ and $Q_{1}$ is a divided prime ideal of $A$ (see Lemma 2.2 (b) and Proposition 2.4 (d)), applying the above upshot of [13, Theorem 4.2 (c)] to the pullback $A=A_{Q_{1}} \times_{\mathrm{k}_{A}}\left(Q_{1}\right) A / Q_{1}$ yields the following conclusion: $A$ is a Bézout domain if and only if $A_{1}$ is a Bézout domain and $A_{Q_{1}}$ is a valuation domain. By (2), $A_{1}$ is a Bézout domain, and so we need only prove that $A_{Q_{1}}$ is a valuation domain. This, in turn, follows since $A$ is a Prüfer domain by Theorem 2.21 [(1) or (2) implies (3)].

REMARK 2.24. (a) In view of the motivation provided by [10], Theorem $2.21[(1) \Rightarrow(3)]$ is an appealing result. It is instructive to consider a direct attempt at proving that $A=\lim A_{n}$ is a Prüfer domain, assuming only that each $A_{n}$ is a Prüfer domain and $\varphi_{m, n}: A_{m} \rightarrow A_{n}$ is surjective for each $m \geq n$ in N. Using the criterion for Prüfer domains that "each nonzero finitely generated ideal is invertible", one can construct a proof, somewhat in the spirit of that of Theorem 2.12, if one is free to use two facts: $\Phi_{n}: A \rightarrow A_{n}$ is surjective for each $n \in \mathrm{N}$; and $Q_{m, n}:=\operatorname{ker}\left(\varphi_{m, n}\right)$ is a divided prime ideal of $A_{m}$ for each $m \geq n$ in $\mathrm{N}$. The first of these "facts" is valid, but the second "fact" was shown in Proposition 2.4 (c) as a result of our riding hypotheses. Thus, we have sketched an alternate proof of Theorem $2.21[(1) \Rightarrow(3)]$. More importantly, we have found a new way to motivate those riding hypotheses, for dividedness of the ideals $Q_{n+1, n}$ allows one to recover $\left\{\left(B_{n}, K_{n}\right): n \in \mathrm{N}\right\}$ as in our riding hypotheses, assuming only that $A_{1}$ is not a field and $\varphi_{n+1, n}$ is not an isomorphism. Indeed, taking $B_{n}:=\left(A_{n+1}\right)_{Q_{n+1, n}}$ and $K_{n}=\mathrm{k}_{A_{n+1}}\left(Q_{n+1, n}\right)$ leads to data satisfying our riding hypotheses. One moral is that both of our approaches to Theorem $2.21[(1) \Rightarrow(3)]$ naturally lead to consideration of divided prime ideals.

(b) Although we have determined when $A$ (under our riding hypotheses) belongs to several important classes of integral domains, additional such results are possible. Analogously to Corollary 2.23, one could use part (b), rather than part (c), of [13, Theorem 4.2] to determine when $A$ is a G-GCD domain (in the sense of [1]). Similarly, [13, Theorem 4.1] can be used to characterize when $A$ is a Prüfer $v$-multiplication domain $(P V M D)$. We leave the details to the interested reader.

In closing, we consider one of the motivations for much of [10] and the above work. Recall (cf. [4]) that an integral domain $D$ is a going-down domain if the inclusion map $D \rightarrow E$ satisfies $G D$ for each integral domain $E$ containing $D$ (equivalently, for each overring $E$ of $D$ ). Many of the aboveconsidered types of integral domains are examples of going-down domains. (For example, locally divided domains and $i$-domains are going-down do- 
mains; hence, so are valuation domains, divided domains, $P^{r} V D s, P V D s$, Prüfer domains, and Bézout domains: a redundant list which corresponds to the order in which the concepts appeared above. On the other hand, a quasiPrüfer domain need not be a going-down domain; the same holds for a GGCD domain and a $P V M D$.) Being a going-down domain is a local property of integral domains (cf. [5]). Any locally divided domain is a going-down domain [5, Remark 2.7 (b)]; the converse holds for integral domains which are seminormal (in the sense of [23]), essentially by the proof of [5, Theorem 2.5].

Proposition 2.25. Consider the following three conditions:

(1) For each $n \in \mathrm{N}, A_{n}$ is a going-down domain;

(2) $A_{1}$ is a going-down domain and, for each $n \in \mathrm{N}, B_{n}$ is a going-down domain;

(3) A is a going-down domain.

Then:

(a) $(3) \Rightarrow(1)$.

(b) Assume that $K_{n}=q f\left(A_{n}\right)$ for each $n \in \mathrm{N}$. Then (1) $\Leftrightarrow$ (2).

(c) Assume that for each $n \in \mathrm{N}, K_{n}=q f\left(A_{n}\right), B_{n}$ is seminormal and $A_{1}$ is seminormal. Then $(1) \Rightarrow(3)$.

(d) Assume that for each $n \in \mathrm{N}, K_{n}=q f\left(A_{n}\right)$ and $B_{n}$ is integrally closed. Then $(1) \Rightarrow(3)$.

Proof. Since any factor domain of a going-down domain is a going-down domain [5, Remark 2.11 and Remark 3.2 (a), (b)], we have that (3) $\Rightarrow(1)$, giving (a). Assume henceforth that $K_{n}=q f\left(A_{n}\right)$ for each $n \in \mathrm{N}$. Then, by translating [7, Corollary 2.3] and applying it to the pullback $A_{n+1}=B_{n} \times_{K_{n}} A_{n}$, we see that $A_{n+1}$ is a going-down domain if and only if both $A_{n}$ and $B_{n}$ are going-down domains. Then (b) follows easily.

(c) Notice that $A_{n+1}$ is seminormal if and only if both $A_{n}$ and $B_{n}$ are seminormal. Then, given (1) and the assumptions in (c), we have that $A_{n}$ is a seminormal going-down domain for each $n \in \mathrm{N}$. By the above remarks, $A_{n}$ is locally divided for each $n \in \mathrm{N}$ and so, by Corollary 2.17 (b), $A$ is a locally divided domain. In particular, $A$ is a going-down domain, yielding (3) and completing the proof of (c).

(d) It is enough to show that $A_{P}$ is a going-down domain for each maximal $P \in \operatorname{Spec}(A)$. By Proposition 2.15 (d), we may suppose $A$ is quasilocal; by Corollary 2.6, $A_{1}$ is also quasilocal (and, by hypothesis, a going-down domain). Recall the following criterion [5, Theorem 2.5]: a quasilocal integral domain $D$ is a going-down domain if and only if $D$ has an integral overring $E$ such that $E$ is a divided domain and the inclusion map $D \rightarrow E$ is an $i$-extension. For $D:=A_{1}$, take $C_{1}$ to be such an $E$. Notice, by integrality, 
that $C_{1}$ is not a field (since $A_{1}$ is not a field). For each $n \in \mathrm{N}$, put $C_{n+1}:=B_{n} \times{ }_{K_{n}} C_{n}$, and consider $C:=\lim C_{n}$. By the above criterion, it suffices to show that $C$ is an integral overring of $A, C$ is a divided domain, and the inclusion map $A \rightarrow C$ is an $i$-extension.

By Lemma 2.11 (d), $C$ is an overring of $A$. (Of course, we may view $A \subseteq C$ since lim preserves injections.) Moreover, by [11, Corollary 1.5 (5)], $C_{n}$ is integral over $A_{n}$, for each $n \in \mathrm{N}$. It follows from the hypotheses that $C_{n}$ is overring of $A_{n}$ for each $n$, and so, by Theorem 2.12, $C \subseteq A^{\prime}$. In particular, $C$ is an integral overring of $A$. Next, notice that for each $n, B_{n}$ is a quasilocal (semi)normal going-down domain and so, by the above comments, it follows from [5] that $B_{n}$ is a divided domain. Hence, by applying [7, Proposition 2.12] to the pullback construction of $C_{n+1}$, we see by induction on $n$ that $C_{n}$ is a divided domain for each $n$. Therefore, by Corollary 2.17 (a), $C$ is a divided domain. Finally, since $A_{n+1}=C_{n+1} \times_{C_{n}} A_{n}$, the topological description of prime spectra of pullbacks [11, Theorem 1.4] gives that $A_{n} \rightarrow C_{n}$ is an $i$ extension for each $n$. Therefore, by Proposition 2.13 (c), $A \rightarrow C$ is an $i$-extension, completing the proof.

\section{REFERENCES}

1. D.D. Anderson and D.F. Anderson, Generalized GCD domains, Comm. Math. Univ. St. Pauli 28 (1979), 215-221.

2. D.F. Anderson and D.E. Dobbs, Pairs of rings with the same prime ideals, Canad. J. Math. 32 (1980), 362-384.

3. J. Coykendall, D.E. Dobbs and B. Mullins, On integral domains with no atoms, Comm. Algebra 27 (1999), 5813-5831.

4. D.E. Dobbs, On going-down for simple overrings, II, Comm. Algebra 1 (1974), 439-458.

5. D.E. Dobbs, Divided rings and going-down, Pacific J. Math. 67 (1976), 353-363.

6. D.E. Dobbs, Fragmented integral domains, Portugal. Math. 43 (1985-1986), 463-473.

7. D.E. Dobbs, On Henselian pullbacks, in D.D. Anderson, ed., Factorization in Integral Domains, Lecture Notes in Pure and Appl. Math. 189 (1997), 317-326.

8. D.E. Dobbs and M. Fontana, Sur les suites dimensionelles et une classe d'anneaux distingués qui les déterminent, C.R. Acad. Sci. Paris A-B 306 (1988), 11-16.

9. D.E. Dobbs, M. Fontana and S. Kabbaj, Direct limits of Jaffard domains and S-domains, Comment. Math. Univ. St. Paul. 39 (1990), 143-155.

10. D.E. Dobbs, M. Fontana and I.J. Papick, Direct limits and going-down,Comment. Math. Univ. St. Paul. 31 (1982), 129-135.

11. M. Fontana, Topologically defined classes of commutative rings, Ann. Mat. Pura Appl. 123 (1980), 331-355.

12. M. Fontana, Sur quelques classes d'anneaux divisés, Rend. Sem. Mat. Fis. Milano 51 (1981), 179-200.

13. M. Fontana and S. Gabelli, On the class group and the local class group of a pullback, J. Algebra 181 (1996), 803-835.

14. M. Fontana, J.A. Huckaba and I.J. Papick, Prüfer Domains, Dekker, New York, 1997.

15. R. Gilmer, Multiplicative Ideal Theory, Dekker, New York, 1972. 
16. A. Grothendieck and J.A. Dieudonné, Eléments de Géometrie Algébrique I,Springer, Berlin, 1971.

17. J.R. Hedstrom and E.G. Houston, Pseudo-valuation domains, Pacific J. Math. 75 (1978), $137-147$.

18. P.J. Hilton and U. Stammbach, A Course in Homological Algebra, Springer, Berlin, 1971.

19. I. Kaplansky, Commutative Rings, rev. ed., Univ. Chicago Press, Chicago, 1974.

20. M. Nagata, Local Rings, Wiley-Interscience, New York, 1962.

21. I.J. Papick, Topologically defined classes of going-down rings, Trans. Amer. Math. Soc. 219 (1976), 1-37.

22. A. Seidenberg, On the dimension theory of rings II, Pacific J. Math. 4 (1954), 603-614.

23. R.G. Swan, On seminormality, J. Algebra 67 (1980), 210-229.

24. A. Wiseman, Integral extensions of linearly compact domains, Comm. Algebra 11 (1983), 1099-1121.

DEPARTMENT OF MATHEMATICS

UNIVERSITY OF TENNESSEE

KNOXVILLE, TN 37996-1300

USA

Email:dobbs@math.utk.edu
DIPARTIMENTO DI MATEMATICA UNIVERSITà DEGLI STUDI ROMA TRE 00146 ROMA

ITALY

Email: fontana@mat.uniroma3.it 